Research and testing laboratories

\title{
World at work: Research and testing laboratories
}

\section{R J Emery, G L Delclos}

\section{Spotlight on a diverse industry}

M anufacturing facilities typically focus on the creation of a finite array of products in large volumes. Consequently, the potential hazards inherent to these workplaces are limited in scope, although they may be high in output. On the other hand, a research and testing laboratory performs testing and diagnostic evaluation of samples, a setting not typically within the production line sequence; hence, the likelihood of exposures to large volumes of potentially hazardous agents is usually much lower. However, the sophisticated analytical procedures conducted in laboratory settings often involve the use of a variety of exotic and potentially hazardous agents. It is this array of hazards, combined with differences among potentially exposed individuals, that makes the laboratory setting a unique working environment.

\section{TASKS OF THE JOB}

Laboratories are ubiquitous in today's world, are designed to fulfil various roles, and can vary markedly in size, from that of a small closet area to multiple floors in large buildings. Commercially operated laboratories provide analytical testing services for products, such as processed food, or are involved in the research and development of new products. Governments operate laboratories for quality control purposes to ensure product integrity and performance. Governments also operate forensic laboratories for the identification of evidence in crimes or to determine cause of death. Healthcare facilities maintain medical, clinical, and/or veterinary laboratories to accommodate the processing of clinical specimens for medical diagnoses, whereas universities house laboratories directed at research endeavours. Based on data from the United States Bureau of Labor Statistics, in 2003 there were more than 430000 persons employed in medical, diagnostic, veterinary, and/or testing laboratories-that is, approximately $0.3 \%$ of the civilian workforce (http://www.bls.gov/oes/2003/may/oessrci. htm).
Regardless of their purpose or setting, all laboratories share certain characteristic tasks. In a broad sense, the tasks inherent to laboratory work include some or all of the following: development of testing protocols and sampling strategies; instrument calibration and internal quality assurance; product sampling and collection; transport and delivery of samples to the laboratory; receipt and recording of sample entry; sample preparation; sample analysis; calculation and reporting of results; and waste disposal. Of these tasks, those of greatest concern for potentially hazardous exposures involve sample collection, preparation, and analysis.

All laboratories perform analyses using equipment and techniques that subject samples to extreme physical, chemical, radiological, and/or biological conditions, often simultaneously. In turn, these procedures represent potentially hazardous exposures to workers in and around these settings. Effective implementation of controls and protective measures for these workers requires an in-depth appreciation of the complexities of the laboratory work environment.

In addition to the various potential hazards that may be present in the laboratory work setting, it is important to consider the diversity of the potential at-risk population. First are the laboratory workers themselves. These individuals are recruited, hired, trained, and receive direct compensation for their efforts; their adherence to accepted work practices can be a condition of continued employment or annual performance evaluations. However, these kinds of administrative controls may not extend to other individuals who can spend time in a laboratory, including students, volunteers, and visiting scholars. Because of the cyclical nature and variable duration of such exchanges and visits, these persons may not be subjected to the same surveillance measures, workplace monitoring, hazard awareness training, safety performance assessment, controls, or expectations afforded to usual laboratory employees.

\section{HAZARDS OF THE JOB AND IN THE WORKPLACE}

The potential hazards present in a laboratory environment can be classified, from a pragmatic standpoint, into four main categories: chemical, physical, radiological, and biological. Each agent class exhibits a unique set of concerns, warranting specialised attention.

\section{Chemical hazards}

Given their large number, a detailed discussion of individual chemical agents used in laboratory settings is beyond the scope of this article. However, analysis of laboratory samples typically involves a series of elaborate processes that allow the isolation of a specific characteristic of a compound. In the processing and analysis of these samples, a wide array of exotic hazardous chemicals may be used, which can be broadly classified by the health and safety risks inherent in their use. These risk categories include carcinogens, toxic or highly toxic agents, reproductive toxins, irritants, corrosives, sensitisers, hepatotoxins, nephrotoxins, neurotoxins, haematopoietic system toxins, and agents which damage the lungs, skin, eyes, or mucous membranes. ${ }^{1}$ The most likely routes of exposure are inhalation and absorption through skin or mucous membranes.

In comparison to private industry as a whole, where trends in the incidence of both work related skin disorders and acute toxic inhalations have been declining steadily since 1998, rates in laboratory settings have either fluctuated or increased (table 1).

\section{Physical hazards}

In addition to the hazards represented by the intrinsic toxicity of a chemical agent, its physical state may also pose a health hazard (for example, combustible liquids, compressed gas, explosive, flammable, pyrophoric compounds, and compounds that are unstable or water reactive)

From an ergonomic standpoint, laboratory analytical techniques can require long periods of standing on hard floors or sitting in one position, together with the performance of repeated tasks, increasing the chances of low back pain and other musculoskeletal discomfort. Work surfaces not matched to the worker's height and inadequate task lighting in the work area can also cause work discomfort. Repetitive procedures such as opening and closing vial caps, pipetting (fig 1), and sample sorting hold the potential to result in repetitive motion injuries or other cumulative trauma. Similar concerns exist when prolonged periods of data entry or other computer based work are required. In selected laboratory settings, such as 
Table 1 Trends in incidence rates* of occupational illness for all private industry and research/testing laboratories, United States, 1998-2001

\begin{tabular}{lcccc}
\hline $\begin{array}{l}\text { Occupational illness } \\
\text { category }\end{array}$ & Year & $\begin{array}{l}\text { All private } \\
\text { industry }\end{array}$ & $\begin{array}{l}\text { Medical and dental } \\
\text { laboratories }\end{array}$ & Testing services \\
\hline Overall & 1998 & 1.9 & 4.3 & 0.2 \\
& 1999 & 1.7 & 11.1 & 2.2 \\
& 2000 & 1.5 & 2.5 & 1.7 \\
& 2001 & 1.6 & 8.1 & 0.6 \\
Skin disorders & 1998 & 6.0 & 4.4 & 2.2 \\
& 1999 & 4.9 & 3.9 & 3.1 \\
& 2000 & 4.6 & 6.7 & 4.3 \\
Toxic inhalations & 2001 & 4.3 & 3.6 & 5.3 \\
& 1998 & 2.0 & & \\
& 1999 & 1.8 & 2.0 & 1.4 \\
& 2000 & 1.6 & 4.9 & 2.5 \\
Physical agents & 2001 & 1.6 & 1.4 & 4.6 \\
& 1998 & 1.9 & 2.9 & 4.2 \\
& 1999 & 1.7 & 4.3 & 0.2 \\
& 2000 & 1.5 & 11.1 & 2.2 \\
Repetitive trauma & 1998 & 28.5 & 2.5 & 1.7 \\
& 1999 & 27.3 & 8.1 & 0.6 \\
& 2000 & 26.3 & 33.0 & 22.8 \\
& 2001 & 23.8 & 35.2 & 25.1 \\
& & 1.6 & 30.7 & 15.1 \\
& & & 42.0 & 22.9 \\
\hline
\end{tabular}

*Cases per 10000 full time employed workers.

Source: US Bureau of Labor Statistics, www.bls.gov.
Lasers have become a common source of non-ionising radiation used in laboratories. Laser systems are classified according to the possible threat they represent to key organ systems such as the eye and skin, ranging from class I, which does not present harm during use, to class IV, which can produce significant eye and/or skin damage. ${ }^{3}$ Other sources of non-ionising radiation in laboratories include radio frequency, microwave, and infrared radiation. Although acute health effects such as burns or localised heating from exposure to non-ionising radiation are well recognised, the possibility of chronic effects is still being evaluated. ${ }^{4}$

\section{Biological hazards}

Potentially infectious agents may be encountered in laboratories, such as microbiology laboratories, when analysing samples derived from humans, plants, or animals, and risks associated with handling such specimens are dependent on the specific microorganism and the pathway of exposure. A hallmark series of elegant surveys conducted many years ago by Sulkin and Pike on laboratory acquired infections showed that laboratory acquired infections paralleled the prevalent infectious diseases at the time, and were most likely transmitted via procedures that resulted in the creation of aerosols. ${ }^{56}$ On the other hand, infectious agents such as human immunodeficiency virus, hepatitis $B$, and hepatitis $C$ viruses are primarily transmitted in the laboratory setting via percutaneous transmission or contact with mucous membranes or non-intact skin; in these cases, the most common vehicles of transmission are needles and cutting sharps. More recently, occupationally acquired infections caused by "new" microorganisms, such as West Nile virus, have been documented in laboratory workers. ${ }^{7}$

\section{Simultaneous exposures}

Compounding the diversity of potential hazards present in the laboratory setting are situations involving simultaneous exposures, which can be commonplace. For example, it is not unusual to encounter a laboratory environment where individuals work in a building (with its concurrent physical hazards), and perform extractions on potentially infectious biological materials using chemical solvents and radioisotope tracers. The appropriate design and implementation of safety measures to control such simultaneous exposure situations warrants a sound understanding of the risks and associated controls for each type of agent on both the part of the worker and the occupational health professionals supporting the operations.
Figure 1 Repetitive tasks, such as pipetting, are common in research and clinical laboratories. that precludes direct accessibility to the radioactive source. 
Thus, a laboratory procedure involving the manipulation of potentially infectious agents may require the use of an enclosure device such as a biological safety cabinet, but if the process involves an ignition source and potentially flammable chemicals with lower vapour pressures, gases could accumulate in the safety cabinet enclosure and cause an explosion. Likewise, personal protective equipment intended for one hazard may not provide adequate protection against a second agent. A classic example would be the use of latex gloves, which provide protection against the transmission of infectious agents through breaks in the skin, but could allow permeation of certain chemicals.

\section{OCCUPATIONAL HEALTH AND SAFETY MANAGEMENT}

The protection of workers in laboratory environments requires a combination of clear management commitment, appropriate facility design, engineering controls, worker training, personal protective equipment, routine surveillance, periodic audits and inspections, and effective communication. Ultimately, though, an effective health and safety programme depends most critically on upper management support and commitment. When upper management clearly articulates its dedication to maintaining a work environment that is safe and healthy, the remaining necessary protective measures become easier to implement.

\section{Facility design and engineering controls}

Central to the protection of workers is proper planning and design of the

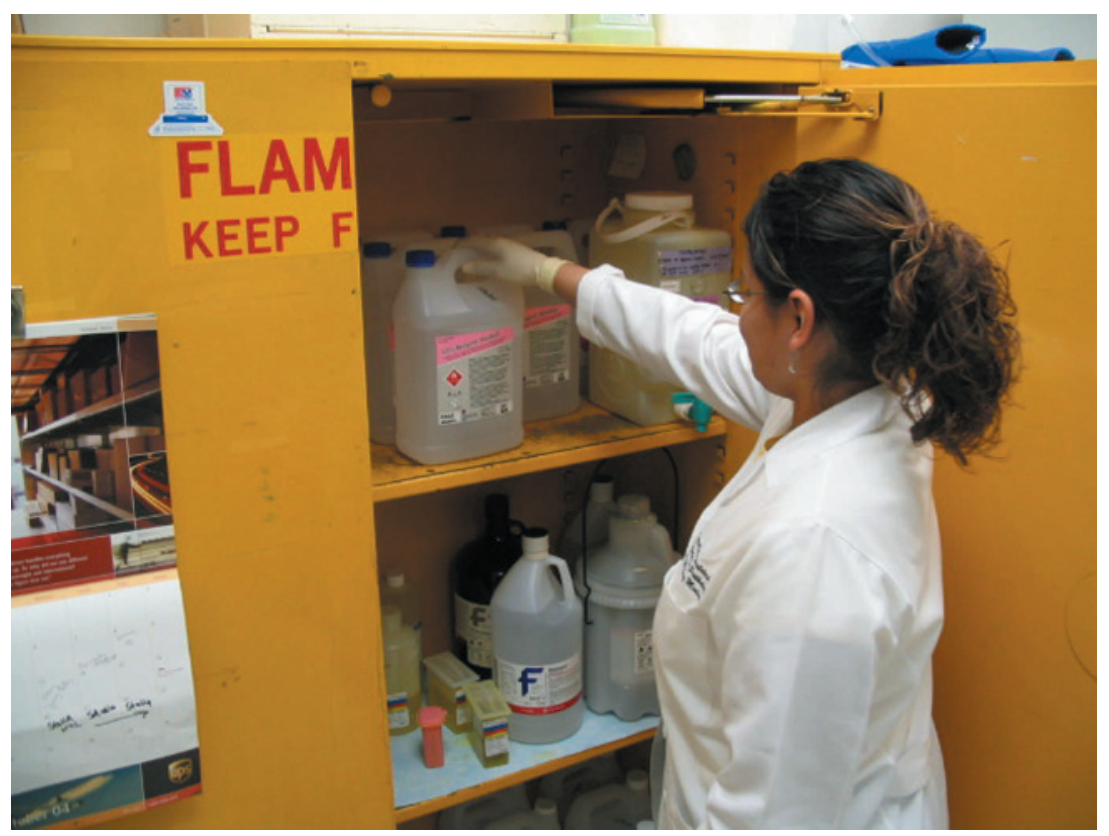

Figure 2 An example of proper storage of hazardous chemicals.

laboratory environment, which has evolved markedly over the past 30 years. The design should be comprehensive, with attention to areas for storage of chemicals (fig 2), general and local exhaust ventilation, adequate workstation design and task lighting (fig 3), implementation of appropriate fume hoods and wash basins, disposal of laboratory waste, and accessible safety eyewash stations and emergency showers. Because laboratories are usually housed within a larger physical structure (either permanently fixed or mobile in nature), structural safety hazard controls such as effective fire detection and suppression, and occupant means of egress are essential. ${ }^{18}$ The presence of sophisticated analytical equipment mandates the availability and proper functioning of electrical power supplies and associated safety features, such as ground fault circuit interrupters. For example, gel electrophoresis units employ high electrical currents to separate chemical compounds, a procedure performed in the presence of electrically conductive liquids, making it essential to incorporate safety systems able to interrupt current flow in the event of a system malfunction. ${ }^{9}$ In settings where flammable or explosive vapours or gases may be present, specialised designs for the enclosure of switches and lighting sources may also be needed.

Facilities should be designed with means of egress in mind, including clear hallways and proper placement of office areas to avoid traversing the laboratory area in the event of an accidental release. Shutoff valves for gases and 


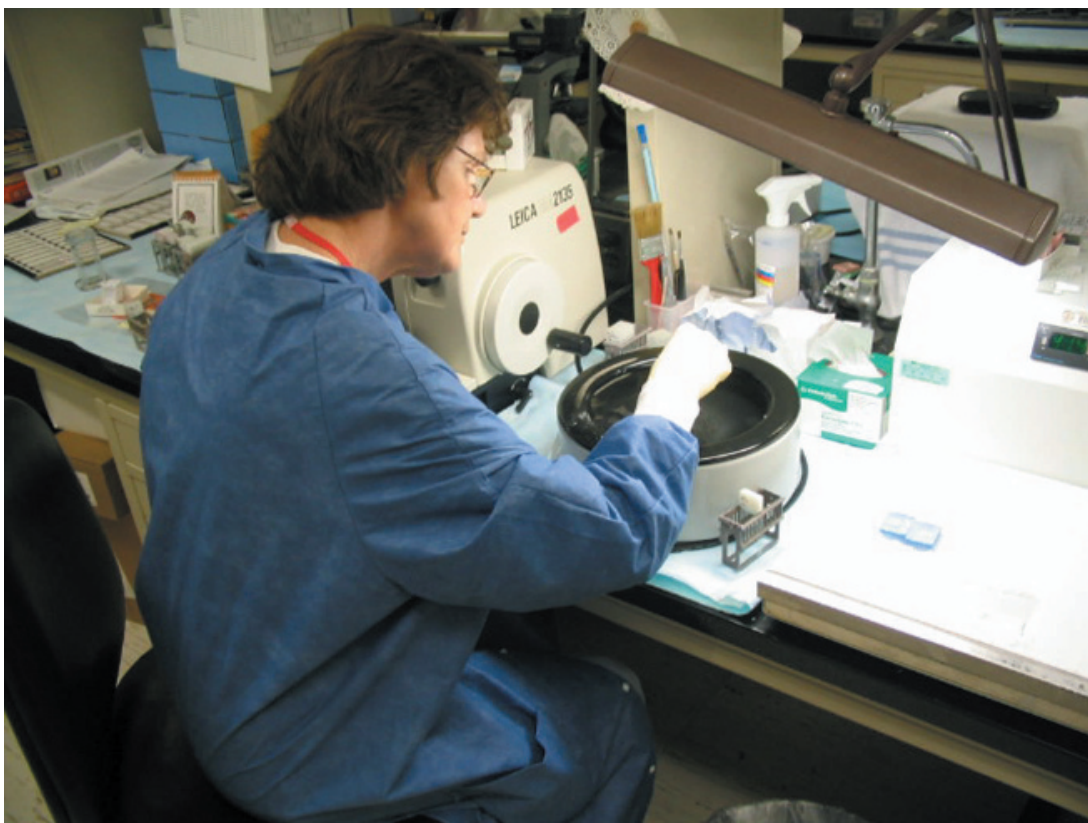

Figure 3 Workstation layout and task lighting are important considerations when designing laboratory facilities.

receive proper training in the identification and safe handling of chemicals and have ready access to information on their hazards. Material safety data sheets and other technical information sheets, often created by chemical product manufacturers, can provide important initial safety information regarding specific risks, and should be readily available in the workplace.

Training of employees in the handling of radioactive sources and biological agents, the latter emphasising the use of universal (standard) precautions, addresses radiological and biological hazards. In the USA, worker training in these areas is mandated under the Nuclear Regulatory Commission and the OSHA Bloodborne Pathogens Standard. ${ }^{13} 14$

\section{Surveillance and safety committees} Because the laboratory environment is inherently dynamic, change is the norm. Therefore, routine health and safety audits and assessments are necessary to determine if any changes have resulted in the emergence of new potential hazards. For example, the placement of a new analytical device in a local exhaust ventilation fume hood could result in the partial block of airflow, producing eddy currents which actually result in reintroduction of contaminants into the work environment (video clip 2). Ideally, such periodic inspections should be conducted by health and safety personnel, with the active involvement of the laboratory staff, thus stimulating local interest and control over the safety systems in place.
One way of establishing a community culture of safety in the organisations hosting laboratory work environments is through the creation of safety committees that are inclusive of representation of upper management and workers. Through the routine reporting of both the successes and failures of an organisation's health and safety efforts, the workforce can be appraised of the systems and procedures that work, and those that warrant attention. The lessons learned from these efforts can be applied to the non-employee populations as well. Active worker involvement, combined with upper management commitment, can represent a powerful tool in maintaining a healthy, safe and productive environment.

\section{CONCLUSION}

Although there are many workplaces in both developed and developing nations that are associated with significant occupational hazards, laboratories represent perhaps one of the microenvironments with the greatest diversity of risks, combined with a diverse at-risk population. The protection of workers in these environments requires an in-depth knowledge of the various exposures (which may occur simultaneously), management support of health and safety, appropriate facility design, worker training, protective equipment, a team approach to surveillance and inspections, and effective communication. When applied appropriately, these measures can assist laboratories in fulfilling their essential functions so that workers remain productive without undue occupational risk.

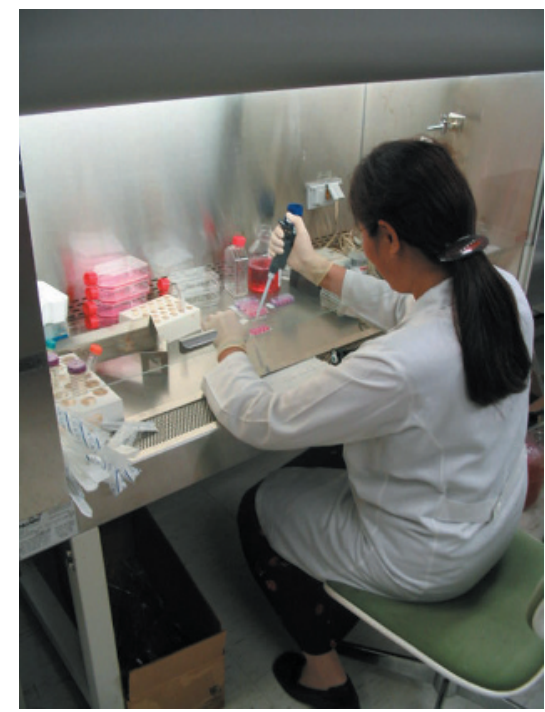

Figure 4 Laboratory worker handling infectious agents under a biological safety cabinet.

Occup Environ Med 2005;62:200-204. doi: 10.1136/oem.2004.015305

\section{Authors' affiliations}

R J Emery, G L Delclos, Southwest Center for Occupational and Environmental Health, The University of Texas School of Public Health at Houston, Houston, Texas, USA

Correspondence to: Dr R J Emery, Associate Professor of Occupational Health, The University of Texas School of Public Health, PO Box 20186, Houston, TX 77225-0186, USA Robert.J.Emery@uth.tmc.edu

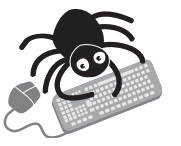

Selected video clips related to this article can be found by visiting the OEM website at www.occenvmed.com/ supplemental.

- Video clip 1: Different types of protective equipment used in laboratories. This video segment shows some examples of safety equipment and devices in a laboratory setting, including eye wash stations and safety showers, flammable storage cabinets, secured compressed gas cylinders, ground fault circuit interrupters, sealed centrifuge cups, sharps containers, hazard signage, and material safety data sheet binders.

- Video clip 2: Air flow through a laboratory fume hood. This video segment illustrates the consequences of improper placement of a piece of laboratory equipment within a local exhaust ventilation fume hood, resulting in the partial block of airflow, producing eddy currents which actually result in reintroduction of contaminants into the work environment. 


\section{REFERENCES}

1 Code of Federal Regulations: Hazard Communication Standard, 29 CFR Sect. 1910.1200 (1994)

2 Mayo RM. Introduction to nuclear concepts for engineers. LaGrange Park, IL: American Nuclear Society, 1998:7.

3 RLI Laser Accident Database [database on the Internet]. Cincinnati, OH: Rockwell Laser Industries. C1996-2004 [cited 2004 Oct 29]. Available from, http://www.rli.com/resources/ accident.asp.

4 National Institute of Environmental Health Sciences (NIEHS). NIEHS report on health effects from exposure to power-line frequency electric and magnetic fields, NIH Publication No.994493. Research Triangle Park NC: National Institute of Environmental Health Sciences, 1999
5 Sulkin SE, Pike RM. Viral infections contracted in the laboratory. N Engl J Med 1949:241:205-13.

6 Sulkin SE, Pike RM. Survey of laboratoryacquired infections. Am J Public Health 1951;41:769-81.

7 Campbell G, Lanciotti R, Bernard B, et al. Laboratory-acquired West Nile virus infections. MMWR 2002;51:1133-5.

8 University of Delaware [homepage on the Internet]. Newark: University of Delaware [cited 2004 May 20]. OHS-Safety Alert-Lab Incident [about 2 screens]. Available from, http://www.udel.edu/OHS/labincident.html.

9 American Industrial Hygiene Association [homepage on the Internet]. Fairfax: American Industrial Hygiene Association [revised 2001 April 26; cited 2004 June 3] Electrical Shock from Electrophoresis Unit.
AlHA Laboratory Health and Safety Committee [about 6 screens]. Available from, http:// www2.umdnj.edu/eohssweb/aiha/accidents/ electrical.htm\#Unit.

10 Sussman GL, Beezhold DH. Allergy to latex rubber. Ann Intern Med 1995; 122:43-6.

11 Fisher AA. Allergic contact reactions in health personnel. J Allergy Clin Immunol 1992;90:729-38.

12 Code of Federal Regulations: Occupational Exposure to Hazardous Chemicals in Laboratories, 29 CFR Sect. 1910.1450 (1990).

13 Code of Federal Regulations: Notices, Instructions and Reports to Workers: Inspection and Investigations, 10 CFR Part 19 (1990)

14 Code of Federal Regulations: Bloodborne Pathogens, 29 CFR Sect. 1910.1030 (1992)

\section{$\mathrm{ECHO}$}

\section{Specific criteria help patients to identify hip pain}

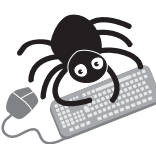

Please visit the Occupational and

Environmental Medicine website [www. occenvmed. com] for a link to the full text of this article.
$\mathrm{R}$ esearchers have come up with a way of defining hip pain that is robust enough to use for selecting patients for population studies. It should ensure more accurate studies and more meaningful results.

They tested their method by asking patients in a primary care practice to respond to a directly worded question whether they had "hip pain" and, again, with a validated diagram defining the location of hip pain. They then compared the three groups of patients with pain (responding positively to the worded question only, the diagram only, and both question and diagram) with patients without hip pain for a range of markers of hip disease. These included consulting a family doctor, taking prescribed painkillers or other remedies, difficulty in walking and use of a stick and-in a subset of each group - the range of hip movement and $x$ ray evidence of hip disease.

Patients with hip pain according to both question and diagram were positive for a greater proportion of the markers of hip disease, including some restricted movement and $x$ ray evidence, than the others with pain and those without pain in age and sex adjusted comparisons. The overall response rate to the postal survey was $75 \%$.

This cross sectional study surveyed 2935 patients aged 18-80 years in a Greater Manchester practice. A consecutive sample of patients with hip pain were age and sex matched to the sample without hip pain.

Absence of a standard for defining hip pain has been a potential limitation in previous research.

A Birrell F, et al. Annals of the Rheumatic Diseases 2005;64:95-98. 